\title{
Energy Spectra and Wave Function Analysis of q-Deformed Modified Poschl-Teller and Hyperbolic Scarf II Potentials Using NU Method and a Mapping Method
}

\author{
A Suparmi $^{1 *}, \mathrm{C} \mathrm{Cari}^{2}, \mathrm{H}$ Yuliani ${ }^{2}$
}

${ }^{1,2}$ Physics Department, Sebelas Maret University, J1. Ir. Sutami no 36A Kentingan Surakarta 57126. Ph. 646994, 636895. Fax.646655, Indonesia

${ }^{3}$ Graduate student, Physics Department, Sebelas Maret University, Jl. Ir. Sutami no 36A Kentingan Surakarta 57126. Ph. 646994, 636895. Fax.646655, Indonesia

*E-mail:suparmiuns@gmail.com

The research is partially financed by Hibah Pascasarjana 2340/UN27.10/PG/2012

\begin{abstract}
The solution of Schrodinger equations for q-deformed modified Poschl-Teller and hyperbolic Scarf II potentials are investigated using Nikiforov-Uvarov method and a mapping method. By applying a translation of spatial variable non-deformed potentials are mapped on to deformed potential or vice versa and as a product the potential's parameters scaling obtained. The bound state energy spectra obtained using NU method are given in the close form and the corresponding wave functions are formulated in terms of the Jacobi Polynomials. The energy spectra and the radial wave functions of the system are also produced by a mapping using potential's parameters scaling from the non-deformed to the deformed potential.
\end{abstract}

Keywords:Energy spectra, wave-function, q-deformed hyperbolic Scarf II, q-deformed modified Poschl-Teller, Nikiforov-Uvarov method, a mapping method

\section{Introduction}

The exact analytical solution of Schrodinger equations for a class of shape invariant potentials are only possible if the the angular momentum $l=0$. For $l \neq 0$, the Schrodinger equation can only be solved approximately by using different suitable approximation scheme for centrifugal term. One of the suitable approximation scheme is firstly proposed by Greene and Aldrich (1976) and recently improved (Akpan et.al. 2012), this approximation works very well for potentials whose functions are hyperbolic or trigonometric function.

Quantum deformation has received much attention because of its relation with applications in nuclei (Sviratcheva 2004; Honusek 1992), statistical-quantum theory and conformal field theory (Swamy 1998; Spiridonov 1992). The q-deformed potential is special kind of f-deformed potential with only one deformed parameter q (Darareh \& Harouni 2011). The q-deformed quantum harmonics oscillator has been investigated intensively by some authors in describing the physical models, such as vibrational and rotational spectra of molecules (Ballesteros, A.,et.al. 2005; Bonatsos 1992). The quantum deformation of hyperbolic potential which was introduced by Arai (1991), has been investigated by some authors (Eshghi, 2012; Akpan et. al. 2012 Eg rifes et. al. 2000).

Dutra (2005) has reinterpreted the idea of quantum deformation as a kind of parameter scaling symmetry of the model, so the q-deformed potential is not a new class of potential. The nondeformed potentials are mapped into the corresponding non-deformed ones or vice-versa by a convenient translation of the spatial variable, and so its energy spectra and wave functions.

In this work we analyze the energy spectra and wave functions of q-deformed modified PoschlTeller and hyperbolic Scarf II potentials. The hyperbolic Scarf II potential is also called as 
Advances in Physics Theories and Applications

ISSN 2224-719X (Paper) ISSN 2225-0638 (Online)

Vol.16, 2013

generalized Poschl-Teller potential (Derezi'nski \& Wrochna 2010). Both potentials have been investigated intensively by some authors (Agboola, 2011; Hassan,et.al. 2012; Ikhdair, 2009; Suparmi, et.al. 2012). We intend to determine the energy spectra and wave functions of the qdeformed potentials using Nikiforov-Uvarov (NU) method and mapping method. The modified Poschl-Teller potential has been used in atom optics and nanostructure physics (Dalarsson 2005).

The NU method, that was developed by Nikiforov-Uvarov (1998), was used to solve Schrodinger equation by reducing it to the hypergeometric type equation by a suitable change of variable. The Nikiforov-Uvarov method has been used to obtain the energy eigenvalues and the corresponding eigenfunctions for a number of physical potentials.

This paper is organized as follows. NU method is briefly reviewed in section 1.1. The quantum deformation of hyperbolic potentials and the mapping method are discussed in section 1.2. The energy spectra and the corresponding wave functions of q-deformed modified Poschl-Teller and hyperbolic Scarf II potentials are found using NU method in sections 2.1 and 2.2. The use of a translation of spatial variable that maps energy spectra and wave functions of non-deformed potentials into deformed ones is discussed in section 3 and brief conclusion is presented in section 4.

\subsection{Review of Nikiforov-Uvarov Method}

The one-dimensional Schrodinger equation of any shape invariant potential can be reduced into hypergeometric or confluent hypergeometric type differential equation by suitable variable transformation (Yasuk F. et. al. 2005; Natanzon 1971). The hypergeometric type differential equation, which can be solved using Nikiforov-Uvarov method is presented as

$\frac{\partial^{2} \psi(s)}{\partial s^{2}}+\frac{\bar{\tau}}{\sigma(s)} \frac{\partial \psi(s)}{\partial s}+\frac{\bar{\sigma}(s)}{\sigma^{2}} \psi(s)=0$

where $\sigma(s)$ and $\bar{\sigma}(s)$ are polynomials at most in the second order, and $\bar{\tau}(s)$ is first order polynomial. Equation (1) can be solved using separation of variable method which is expressed as

$\psi=\phi(s) y(s)$

By inserting equation (2) into equation (1) we get hypergeometric type equation as

$\sigma \frac{\partial^{2} y(s)}{\partial s^{2}}+\tau \frac{\partial y(s)}{\partial s}+\lambda y(s)=0$

and $\phi(s)$ is a logarithmic derivative whose solution is obtained from condition

$\frac{\phi^{\prime}}{\phi}=\frac{\pi}{\sigma}$

where the function $\pi(s)$ and the parameter $\lambda$ are defined as

$\pi=\left(\frac{\sigma^{\prime}-\bar{\tau}}{2}\right) \pm \sqrt{\left(\frac{\sigma^{\prime}-\bar{\tau}}{2}\right)^{2}-\bar{\sigma}+k \sigma}$

$\lambda=k+\pi$

The value of $\mathrm{k}$ in equation (5) can be found from the condition that under the square root of equation (5) have to be square of polynomial which is mostly first degree polynomial and therefore the discriminate of the quadratic expression is zero. A new eigenvalue of equation (3) is

$\lambda=\lambda_{n}=-n \tau^{\prime}=\frac{n(n-1)}{2{ }^{2}} \sigma^{\prime \prime}, n=0,1,2$
where $\tau=\tau+2 \pi^{2}$

The new energy eigenvalue is obtained using equation (6) and (7). 
To generate the energy eigenvalues and the corresponding eigenfunctions, the condition that $\tau^{\prime}<0$ is required. The solution of the second part of the wave function, $y_{n}(s)$, is connected to Rodrigues relation which is given as

$y_{n}(s)=\frac{c_{n}}{\rho(s)} \frac{d^{n}}{d s^{n}}\left(\sigma^{n}(s) \rho(s)\right)$

where $C_{n}$ is normalization constant, and the weight function $\rho(s)$ satisfies the condition

$\frac{\partial(\sigma \rho)}{\partial s}=\tau(s) \rho(s)$

The wave function of the system is therefore obtained from equation (4) and (9).

1.2.Review of q-deformed hyperbolic potential and a mapping between q-deformed potential and non-deformed ones

The q-deformed hyperbolic functions introduced by Arai are given by

$\sinh _{q} \alpha r=\frac{\left(e^{\alpha r}-q e^{-\alpha r}\right)}{2} ; \quad \cosh _{q} \alpha r=\frac{\left(e^{\alpha r}+q e^{-\alpha r}\right)}{2} ; \quad \tanh _{q} \alpha r=\frac{\sinh _{q} \alpha r}{\cosh _{q} \alpha r}$

$\sec _{q}=\frac{1}{\cosh _{q} \alpha r} ; \quad \cosh _{q}^{2} \alpha r-\sinh _{q}^{2} \alpha r=q \quad 1-\tanh _{q}^{2} \alpha r=q \sec h_{q}^{2} \alpha r$

By using a translation of spatial variable introduced by Dutra

$$
r=y+\frac{\ln \sqrt{q}}{\alpha}, \quad \quad y=r-\frac{\ln \sqrt{q}}{\alpha}
$$

the deformed hyperbolic functions change into non-deformed ones and vice-versa as follows

$\sinh _{q} \alpha r=\sqrt{q} \sinh \alpha y ; \quad \cosh _{q} \alpha r=\sqrt{q} \cosh \alpha y ; \quad \tanh _{q} \alpha r=\tanh \alpha y$

and $\quad \sinh \alpha y=\frac{\sinh _{q} \alpha r}{\sqrt{q}} ; \quad \cosh \alpha y=\frac{\cosh _{q} \alpha r}{\sqrt{q}} ; \tanh \alpha y=\tanh _{q} \alpha r$

Two of a class of hyperbolic potentials that will be discussed in this paper are the q-deformed modified Poschl-Teller and hyperbolic Scarf II potentials with centrifugal term whose effective potentials, respectively, are expressed as

$V_{e e_{q}}=\frac{\hbar^{2}}{2 \mu}\left(\frac{\kappa(\kappa-1)+l(l+1)}{\gamma^{2} \sinh _{q}{ }^{2}\left(\frac{r}{\gamma}\right)}-\frac{\lambda(\lambda+1)}{\gamma^{2} \cosh _{q}{ }^{2}\left(\frac{r}{\gamma}\right)}+\frac{l(l+1) d_{0}}{\gamma^{2}}\right) \quad$ and $\quad V_{e f_{q}}=\frac{\hbar^{2}}{2 \mu} \alpha^{2}\left(\frac{b^{2}+a(a+1)+l(l+1)}{\sinh _{q}{ }^{2} \alpha r}-\frac{2 b\left(a+\frac{1}{2}\right) \cosh _{q} \alpha r}{\sinh _{q}{ }^{2} \alpha r}+\frac{l(l+1) d_{0}}{\gamma^{2}}\right)$

By using equations (12) and (13) the effective potentials in equation (15) change into

$V_{\epsilon_{q}}=\frac{\hbar^{2}}{2 \mu}\left(\frac{\kappa(\kappa-1)+l(l+1)}{\gamma^{2} q \sinh ^{2}\left(\frac{r}{\gamma}\right)}-\frac{\lambda(\lambda+1)}{\gamma^{2} q \cosh ^{2}\left(\frac{r}{\gamma}\right)}+\frac{l(l+1) d_{0}}{\gamma^{2}}\right) \quad$ and $\quad V_{e_{q}}=\frac{\hbar^{2}}{2 \mu} \alpha^{2}\left(\frac{b^{2}+a(a+1)+l(l+1)}{q \sinh ^{2} \alpha r}-\frac{2 b\left(a+\frac{1}{2}\right) \cosh \alpha r}{\sqrt{q} \sinh ^{2} \alpha r}+l(l+1) d_{0}\right)$

Non-deformed Hyperbolic Poschl-Teller and Scarf II potentials with centrifugal term are expressed as

$V_{e_{q}}=\frac{\hbar^{2}}{2 \mu}\left(\frac{\kappa(\kappa-1)+l(l+1)}{\gamma^{2} \sinh ^{2}\left(\frac{r}{\gamma}\right)}-\frac{\lambda(\lambda+1)}{\gamma^{2} \cosh ^{2}\left(\frac{r}{\gamma}\right)}+\frac{l(l+1) d_{0}}{\gamma^{2}}\right) \quad$ and $\quad V_{\epsilon_{q}}=\frac{\hbar^{2}}{2 \mu} \alpha^{2}\left(\frac{b^{2}+a(a+1)+l(l+1)}{\sinh ^{2} \alpha r}-\frac{2 b\left(a+\frac{1}{2}\right) \cosh \alpha r}{\sinh ^{2} \alpha r}+l(l+1) d_{0}\right)$

By comparing equations (16a) and (16b) we obtain the relation of potential's parameters between the deformed potentials and the corresponding non-deformed potentials. This relation is called as 
potential's parameter scaling. By using the potential's parameter scaling the energy spectrum and wave function of non-deformed are mapped to the deformed ones.

\section{Solution of deformed potential using NU method}

2.1. Solution of Schrodinger equation for q-deformed modified Poschl-Teller with centrifugal term using NU method

The radial Schrodinger equation of q-deformed modified Poschl-Teller with centrifugal term is given as

$\frac{1}{r^{2}} \frac{\partial}{\partial r}\left(r^{2} \frac{\partial R}{\partial r}\right)-\frac{1}{\gamma^{2}}\left(\frac{\kappa(\kappa-1)}{\sinh _{q}{ }^{2} \frac{r}{\gamma}}-\frac{\lambda(\lambda+1)}{\cosh _{q}{ }^{2} \frac{r}{\gamma}}\right) R+\frac{2 M}{\hbar^{2}} E R-\frac{l(l+1)}{r^{2}} R=0$

For $\frac{r}{\gamma} \ll<$ the centrifugal term is approximated by, $\frac{1}{r^{2}} \cong \frac{1}{\gamma^{2}}\left(d_{0}+\sinh _{q}^{-2}\left(\frac{r}{\gamma}\right)\right)$, with $d_{0}=\frac{1}{12}$, and by setting $R(r)=\frac{\chi(r)}{r} ; \varepsilon^{2}=-\frac{2 m}{\hbar^{2}} E$, equation (17) becomes

$\frac{d^{2} \chi(r)}{d r^{2}}-\left\{\frac{\kappa(\kappa-1)+l(l+1)}{\gamma^{2} \sinh _{q}{ }^{2}\left(\frac{r}{\gamma}\right)}-\frac{\lambda(\lambda+1)}{\gamma^{2} \cosh _{q}{ }^{2}\left(\frac{r}{\gamma}\right)}+\varepsilon^{2}+\frac{l(l+1) d_{0}}{\gamma^{2}}\right\} \chi(r)=0$

Equation (18) is solved using NU method by introducing the new variable

$\cosh _{q}{ }^{2}\left(\frac{r}{\gamma}\right)=s$

we have

$s(s-q) \frac{\partial^{2} \chi}{\partial s^{2}}+\left(s-\frac{q}{2}\right) \frac{\partial \chi}{\partial s}-\left\{\frac{\kappa(\kappa-1)+l(l+1)}{4 s(s-q)} s-\frac{\lambda(\lambda+1)}{4 s(s-q)}(s-q)+\left(\frac{\gamma^{2} \varepsilon^{2}}{4}+\frac{l(l+1) d_{0}}{4}\right) \frac{s(s-q)}{s(s-q)}\right\} \chi=0$

By comparing equations (1) and (20) we have

$\sigma=\left(s^{2}-s q\right) ; \bar{\tau}=s-\frac{q}{2}$

$\vec{\sigma}=-\left[\frac{\left\{\kappa(\kappa-1)+l(l+1)-\lambda(\lambda+1)-\left(\gamma^{2} \varepsilon^{2}+l(l+1) d_{0}\right) q\right\} s}{4}+\frac{[\lambda(\lambda+1)] q}{4}+\frac{\left(\gamma^{2} \varepsilon^{2}+l(l+1) d_{0}\right)}{4} s^{2}\right]$

Using equations (5) and (21) we obtain

$\pi=\left(\frac{s-\frac{q}{2}}{2}\right) \pm \sqrt{\left[\frac{\left\{\kappa(\kappa-1)+l(l+1)-\lambda(\lambda+1)-\left(\gamma^{2} \varepsilon^{2}+l(l+1) d_{0}+4 k+1\right) q\right\} s}{4}+\frac{[\lambda(\lambda+1)] q+\frac{q^{2}}{4}}{4}+\frac{\left(\gamma^{2} \varepsilon^{2}+l(l+1) d_{0}\right)+1+4 k}{4} s^{2}\right]}$

The quadratic expression under the square root of equation (22) must be completed square of first degree polynomial therefore equation (22) can be rewritten as

$\pi=\left(\frac{s-\frac{q}{2}}{2}\right) \pm \frac{1}{2} \sqrt{\left(\gamma^{2} \varepsilon^{2}+l(l+1) d_{0}+1+4 k\right)}\left(s+\frac{\left\{\kappa(\kappa-1)+l(l+1)-\lambda(\lambda+1)-\left(\gamma^{2} \varepsilon^{2}+l(l+1) d_{0}+4 k+1\right) q\right\}}{2\left(\left(\gamma^{2} \varepsilon^{2}+l(l+1) d_{0}\right)+1+4 k\right)}\right)$

and the discriminate of the quadratic expression under the square root that must be zero is given as

$\left[\left\{\kappa(\kappa-1)+l(l+1)-\lambda(\lambda+1)-\left(\gamma^{2} \varepsilon^{2}+l(l+1) d_{0}+4 k+1\right) q\right\}\right]^{2}-4\left\{\left(\left(\gamma^{2} \varepsilon^{2}+l(l+1) d_{0}\right)+1+4 k\right)\right\}\left\{[\lambda(\lambda+1)] q+\frac{q^{2}}{4}\right\}=0$

To calculate the value of $\mathrm{k}$ in equation (24), we simplify equation (24) by setting

$\left(\kappa(\kappa-1)+l(l+1)+\frac{q}{4}\right)=v, \lambda(\lambda+1)=t, \gamma^{2} \varepsilon^{2}+l(l+1) d_{0}+1=u$

so we have $16 k^{2} q^{2}-8 k q(v+t-u q)+(v-t-u q)^{2}-4 u q t=0$ 
and gives

$k_{1}=\frac{(\sqrt{v}+\sqrt{t})^{2}-u q}{4 q}=\frac{\left((o+w)^{2}-u\right)}{4} \quad$ and $\quad k_{2}=\frac{(\sqrt{v}-\sqrt{t})^{2}-u q}{4 q}=\frac{\left((o-w)^{2}-u\right)}{4}$

with $o=\sqrt{\frac{v}{q}} ; w=\sqrt{\frac{t}{q}}$

From equations (23), (25) (27), and (28) and by imposing that $\tau^{\prime}<0$ we obtain

$\pi_{1}=-\frac{1}{2} s(w+o-1)+\frac{w q}{2}-\frac{q}{4}$ for $\mathrm{k}_{1} \quad$ or $\quad \pi_{2}=-\frac{1}{2} s(w-o-1)+\frac{w q}{2}-\frac{q}{4}$ for $\mathrm{k}_{2}$

By using equation (8) together with equations (21a) and (29) we have

$\tau_{1}=-s(w+o-2)+w q-q$ for $\mathrm{k}_{1}$ or $\tau_{2}=-s(w-o-2)+w q-q$ for $\mathrm{k}_{2}$

By using equations (6), (27) and (29) we get

$\lambda_{1}=-\frac{u}{4}+\frac{(w+o)^{2}}{4}-\frac{1}{2}(w+o-1)$ for $\mathrm{k}_{1} \quad$ or $\quad \lambda_{2}=-\frac{u}{4}+\frac{(w-o)^{2}}{4}-\frac{1}{2}(w-o-1)$ for $\mathrm{k}_{2}$

The new energy eigenvalue in equation (3) is obtained from equations (7), (21a), and (30) given as

$\lambda=\lambda_{n 1}=w n+o n-n-n^{2}$ for $\mathrm{k}_{1}$ and $\lambda=\lambda_{n 2}=w n-o n-n-n^{2}$ for $\mathrm{k}_{2}$

By equating equations (31) and (32) we get

$u-1=(w+o-2 n-1)^{2}$ for $\mathrm{k}_{1}$ and $u-1=(w-o-2 n-1)^{2}$ for $\mathrm{k}_{2}$

The energy eigenvalue calculated from equations (25) and (33) is given as

$E_{n 1}=-\frac{\hbar^{2}}{2 \mu \gamma^{2}}\left\{\left(\sqrt{\frac{1}{4}+\frac{\lambda(\lambda+1)}{q}}+\sqrt{\frac{1}{4}+\frac{\kappa(\kappa-1)+l(l+1)}{q}}-2 n-1\right)^{2}-l(l+1) d_{0}\right\}$ for $\mathrm{k}_{1}$

$E_{n 2}=-\frac{\hbar^{2}}{2 \mu \gamma^{2}}\left\{\left(\sqrt{\frac{1}{4}+\frac{\lambda(\lambda+1)}{q}}-\sqrt{\frac{1}{4}+\frac{\kappa(\kappa-1)+l(l+1)}{q}}-2 n-1\right)^{2}-l(l+1) d_{0}\right\}$ for $\mathrm{k}_{2}$

From the two equations of energy spectra expressed in equations (34a) and (34b) only equation (34b) that produces the original energy spectrum of modified Poschl-Teller potential, which is obtained for $q=1$ and $l=0$, given as

$E_{2}=-\frac{\hbar^{2}}{2 \mu \gamma^{2}}(\lambda-\kappa-2 n)^{2}$

therefore the values of $k, \pi, \tau, \lambda, \lambda_{n}$ that satisfy the system are $k_{2}, \pi_{2}, \tau_{2}, \lambda_{2}, \lambda_{n 2}$.

The first part of the wave function is obtained from equations (4), (21) and (29) given as

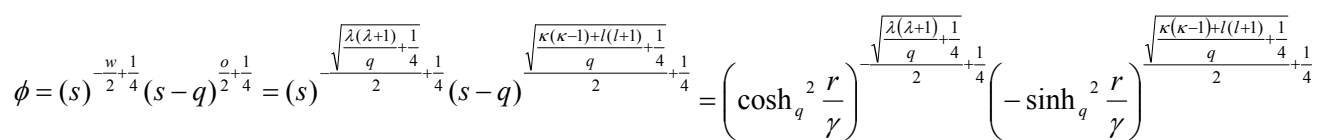

The weight function of the second part of the wave function obtained from equations (10), (21a) and (30) is

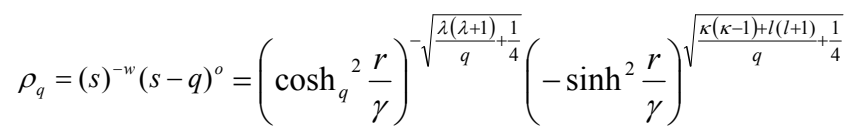

By using equation (9) together with equation (37) we obtain the second part of the wave function as

$y_{n}(s)=\frac{c_{n}}{s^{-w}(s-q)^{o}} \frac{d^{n}}{d s^{n}}\left(s^{-w+n}(s-q)^{o+n}\right)$

by introducing new variable $\frac{s}{q}=x$ and $x=\frac{1+z}{2}$ in equation (38) we get 


$$
y_{n}(s)=\frac{c_{n}(-1)^{n} q^{n}}{x^{-w}(1-x)^{o}} \frac{d^{n}}{d x^{n}}\left(x^{-w+n}\left(1-x^{o+n}\right)=C_{n} n ! q^{n} P_{n}^{(-w, o)}(z)\right.
$$

The total radial wave function of q-deformed modified Poschl-Teller potential with centrifugal term is given as

$$
\chi(r)=\chi(s)=(s)^{-\frac{w}{2}+\frac{1}{4}}(s-q)^{\frac{o}{2}+\frac{1}{4}} y_{n}(s)
$$

$q=1, l=0$ and $n=0$ is the special case where the wave function of the system reduces to the ground state wave function of modified Poschl-Teller potential given as

$$
\chi(r)=\chi(s)=(s)^{\frac{-\left(\lambda+\frac{1}{2}\right)}{2}+\frac{1}{4}(s-q)^{\frac{\left(x-\frac{1}{2}\right)}{2}+\frac{1}{4}}}=\left(\cosh \frac{r}{\gamma}\right)^{-\lambda}\left(-\sinh ^{2} \frac{r}{\gamma}\right)^{\frac{\kappa}{2}}
$$

This result is in agreement with the result determined using hypergeometric equation (Suparmi 2011).

2. 2 The solution of Radial Schrodinger equation for hyperbolic Scarf II potential with centrifugal term

The radial Schrodinger equation for hyperbolic Scarf II potential with centrifugal term is given as

$\frac{1}{r^{2}} \frac{d}{d r}\left(r^{2} \frac{d R}{d r}\right)-\alpha^{2}\left(\frac{b^{2}+a(a+1)}{\sinh _{q}{ }^{2} \alpha r}-\frac{2 b\left(a+\frac{1}{2}\right) \cosh _{q} \alpha r}{\sinh _{q}{ }^{2} \alpha r}\right) R-\frac{l(l+1)}{r^{2}} R+\frac{2 m}{\hbar^{2}} E R=0$

By setting the new wave function ${ }_{R=\frac{\psi(r)}{r}}$ into equation (42) we get

$\frac{d^{2} \psi}{d r^{2}}-\alpha^{2}\left(\frac{b^{2}+a(a+1)}{\sinh _{q}{ }^{2} \alpha r}-\frac{2 b\left(a+\frac{1}{2}\right) \cosh _{q} \alpha r}{\sinh _{q}{ }^{2} \alpha r}\right) \psi+\frac{2 M}{\hbar^{2}} E \psi-\frac{l(l+1)}{r^{2}} \psi=0$

For $\alpha r \ll<1$ the approximation value of centrifugal term is given as $\frac{1}{r^{2}} \cong \alpha^{2}\left(d_{0}+\frac{1}{\sinh _{q}{ }^{2} \alpha r}\right)$ with $d_{0}=\frac{1}{12}$ by substituting it in equation (44) and if $\frac{2 M}{\hbar^{2}} E=-\varepsilon^{2}$ we have

$\frac{d^{2} \psi}{d r^{2}}-\alpha^{2}\left(\frac{b^{2}+a(a+1)+l(l+1)}{\sinh _{q}{ }^{2} \alpha r}-\frac{2 b\left(a+\frac{1}{2}\right) \cosh _{q} \alpha r}{\sinh _{q}{ }^{2} \alpha r}+l(l+1) d_{0}+\frac{\varepsilon^{2}}{\alpha^{2}}\right) \psi=0$

By using a canonical transformation, $\cosh _{q} \alpha r=s$ in equation (44), we obtain

$\left(s^{2}-q\right) \frac{d^{2} \psi(s)}{d r^{2}}+s \frac{d \psi(s)}{d s}-\left(\frac{b^{2}+a(a+1)+l(l+1)}{s^{2}-q}-\frac{2 b\left(a+\frac{1}{2}\right) s}{s^{2}-q}+l(l+1) d_{0}+\frac{\varepsilon^{2}}{\alpha^{2}}\right) \psi(s)=0$

By comparing equation (45) with equation (1) we get

$$
\bar{\tau}=s, \sigma=s^{2}-q, \bar{\sigma}=-\left\{b^{2}+a(a+1)+l(l+1)-2 b\left(a+\frac{1}{2}\right) s+\frac{\left(s^{2}-q\right)\left(\alpha^{2} l(l+1) d_{0}+\varepsilon^{2}\right)}{\alpha^{2}}\right\}
$$

Inserting equation (46) into equation (5) we have

$$
\pi=\frac{s}{2} \pm \sqrt{b^{2}+a(a+1)+l(l+1)-q \frac{\alpha^{2} l(l+1) d_{0}+\varepsilon^{2}}{\alpha^{2}}-k q-2 b\left(a+\frac{1}{2}\right) s+\left(\frac{\alpha^{2} l(l+1) d_{0}+\varepsilon^{2}}{\alpha^{2}}+k+\frac{1}{4}\right) s^{2}}
$$

Since the expression under the square root in equation (47) is quadratic expression with variable $\mathrm{s}$ and it has to be square of first degree polynomial, then equation (47) becomes 
$\pi=\frac{s}{2} \pm \sqrt{\frac{\alpha^{2} l(l+1) d_{0}+\varepsilon^{2}}{\alpha^{2}}+k+\frac{1}{4}}\left(s-\frac{2 b\left(a+\frac{1}{2}\right)}{2\left(\frac{\alpha^{2} l(l+1) d_{0}+\varepsilon^{2}}{\alpha^{2}}+k+\frac{1}{4}\right)}\right)$

and the discriminate of the quadratic expression under the square root that has to be zero is $\left(2 b\left(a+\frac{1}{2}\right)\right)^{2}-4\left(\frac{\alpha^{2} l(l+1) d_{0}+\varepsilon^{2}}{\alpha^{2}}+k+\frac{1}{4}\right)\left(b^{2}+a(a+1)+l(l+1)-q \frac{\alpha^{2} l(l+1) d_{0}+\varepsilon^{2}}{\alpha^{2}}-k q\right)=0$

By setting

$\frac{\alpha^{2} l(l+1) d_{0}+\varepsilon^{2}}{\alpha^{2}}+\frac{1}{4}=v^{2} ; \quad t^{2}=b^{2}+a(a+1)+l(l+1)+\frac{1}{4} q, \quad u^{2}=\left(b\left(a+\frac{1}{2}\right)\right)^{2}, v^{2}+k=p^{2}$

in equation (49) the we get

$k^{2} q-k\left(t^{2}-2 q v^{2}\right)-\left(v^{2}\right)\left(t^{2}-q v^{2}\right)+u^{2}=0$

The value of $\mathrm{k}$ obtained from equation (51) are

$k_{1}=\frac{\left(t^{2}-2 q v^{2}\right)+\sqrt{t^{4}-4 q u^{2}}}{2 q}$ and $k_{2}=\frac{\left(t^{2}-2 q v^{2}\right)-\sqrt{t^{4}-4 q u^{2}}}{2 q}$

By imposing that $\tau^{\prime}<0$ then equation (48) is rewritten as

$\pi_{1}=-s\left(\sqrt{\frac{t^{2}+\sqrt{t^{4}-4 q u^{2}}}{2 q}}-\frac{1}{2}\right)+\frac{b\left(a+\frac{1}{2}\right)}{\sqrt{\left(\frac{t^{2}+\sqrt{t^{4}-4 q u^{2}}}{2 q}\right)}} \quad$ for $\mathrm{k}_{1} \quad$ and $\quad \pi_{2}=-s\left(\sqrt{\frac{t^{2}-\sqrt{t^{4}-4 q u^{2}}}{2 q}}-\frac{1}{2}\right)+\frac{b\left(a+\frac{1}{2}\right)}{\sqrt{\left(\frac{t^{2}-\sqrt{t^{4}-4 q u^{2}}}{2 q}\right)}}$ for $\mathrm{k}_{2}$

By using equations (8) and (53) we get

$\tau_{1}=-2 s\left(\sqrt{\frac{t^{2}+\sqrt{t^{4}-4 q u^{2}}}{2 q}}-1\right)+\frac{2 b\left(a+\frac{1}{2}\right)}{\sqrt{\left(\frac{t^{2}+\sqrt{t^{4}-4 q u^{2}}}{2 q}\right)}} \quad$ for $\mathrm{k}_{1}$ and $\quad \tau_{2}=-2 s\left(\sqrt{\frac{t^{2}-\sqrt{t^{4}-4 q u^{2}}}{2 q}}-1\right)+\frac{2 b\left(a+\frac{1}{2}\right)}{\sqrt{\left(\frac{t^{2}-\sqrt{t^{4}-4 q u^{2}}}{2 q}\right)}}$ for $\mathrm{k} 2$

and from equations (52) and (53) we have

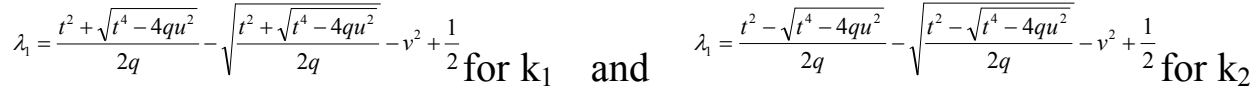

The new eigen value is obtained from equations (46) and (54) as

$\lambda_{1}=\lambda_{n 1}=2 n \sqrt{\frac{t^{2}+\sqrt{t^{4}-4 q u^{2}}}{2 q}}-n-n^{2}$ for $\mathrm{k}_{1}$ and $\lambda_{2}=\lambda_{n 2}=2 n \sqrt{\frac{t^{2}-\sqrt{t^{4}-4 q u^{2}}}{2 q}}-n-n^{2}$ for $\mathrm{k}_{2}$

To simplify the calculation we set

$\frac{t^{2}+\sqrt{t^{4}-4 q u^{2}}}{2 q}=\zeta_{+}^{2}$ and $\frac{t^{2}-\sqrt{t^{4}-4 q u^{2}}}{2 q}=\zeta_{-}^{2}$

and therefore from equations (59), (60), and (61) we get

$E_{n 1}=-\frac{\hbar^{2}}{2 m}\left(\alpha^{2}\left(\zeta_{+}-\eta-\frac{1}{2}\right)^{2}-\alpha^{2} l(l+1) d_{0}\right)$ for $\mathrm{k}_{1}$ and $E_{n 2}=-\frac{\hbar^{2}}{2 m}\left(\alpha^{2}\left(\zeta_{-}-\eta-\frac{1}{2}\right)^{2}-\alpha^{2} l(l+1) d_{0}\right)$ for $\mathrm{k}_{2}$

When we set $\mathrm{q}=1$ and $l=0$ in equations (57) and (58), the energy eigenvalue in equation (58) reduces to the energy eigenvalue of hyperbolic Scarf II potential given as

$$
E_{n 1}=-\frac{\hbar^{2}}{2 m}\left(\alpha^{2}\left(b-n-\frac{1}{2}\right)^{2}\right) \quad \text { or } \quad E_{n 2}=-\frac{\hbar^{2}}{2 m}\left(\alpha^{2}(a-n)^{2}\right)
$$

The energy eigenvalue that satisfies the energy of hyperbolic Scarf II is $E_{n_{2}}$ therefore the values of $k, \pi, \tau, \lambda$ and $\lambda_{n}$ are $k_{2}, \pi_{2}, \tau_{2}, \lambda_{2}$ and $\lambda_{n 2}$. Thus the energy eigenvalue of the system, $E_{n_{2}}$ is 
$E_{n 2}=-\frac{\hbar^{2}}{2 m}\left[\alpha^{2}\left(-\sqrt{\frac{\left\{b^{2}+\left(a+\frac{1}{2}\right)^{2}+\frac{l(l+1)}{4}+\frac{1}{4} q\right\}-\sqrt{\left\{b^{2}+\left(a+\frac{1}{2}\right)^{2}+\frac{l(l+1)}{4}+\frac{1}{4} q\right\}^{2}-4 q b^{2}\left(a+\frac{1}{2}\right)^{2}}}{2 q}}+n+\frac{1}{2}\right)^{2}-\alpha^{2} l(l+1) d_{0}\right]$

The radial wave function is determined using equations (4), (9), (10), (48), (53), and (54).

The first part of the wave function obtained from equations (4), (48) and (53) is

$\phi=(s-\sqrt{q})^{-\frac{1}{2}\left(\zeta_{-}-\frac{1}{2}\right)+\frac{b\left(a+\frac{1}{2}\right)}{2 \zeta_{-} \sqrt{q}}}(\sqrt{q}+s)^{-\frac{1}{2}\left(\zeta_{-}-\frac{1}{2}\right)-\frac{b\left(a+\frac{1}{2}\right)}{2 \zeta_{-} \sqrt{q}}}$

The weight function of the second part of the wave function is obtained by inserting equations (46) and (54b) into equation (10) given as

$\rho=(s-\sqrt{q})^{-\zeta_{-}+\frac{b\left(a+\frac{1}{2}\right)}{\zeta_{-} \sqrt{q}}}(\sqrt{q}+s)^{-\zeta_{-}-\frac{b\left(a+\frac{1}{2}\right)}{\zeta_{-} \sqrt{q}}}$

The second part of the radial wave function obtained from equations (9) and (62) is given as

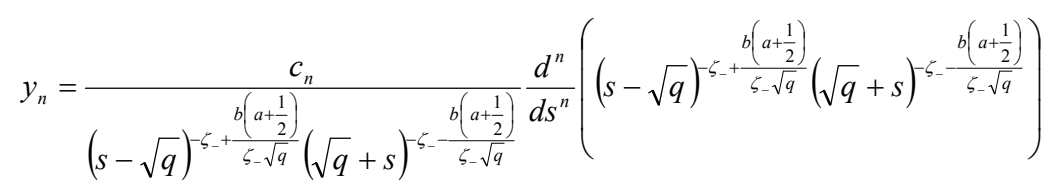

The radial wave function obtained from equations (61) and (63) is given as

$$
\begin{aligned}
& \frac{R(r)}{r}=\psi(r)=\psi(s)=(s-\sqrt{q})^{\frac{1}{2}}\left(-\zeta_{-}+\frac{b\left(a+\frac{1}{2}\right)}{2 \zeta_{-} \sqrt{q}}\right)+\frac{1}{4}(\sqrt{q}+s)^{\frac{1}{2}}\left(-\zeta_{-}-\frac{b\left(a+\frac{1}{2}\right)}{2 \zeta_{-} \sqrt{q}}\right)+\frac{1}{4} y_{n}(s) \\
& =(-1)^{\frac{1}{2} \mu+\frac{1}{4}} C_{n} 2^{n} n !(\sqrt{q})^{\frac{1}{2} \mu+\frac{1}{2} v+\frac{1}{2}+n}(1-x)^{\frac{1}{2} \mu+\frac{1}{4}}(1+x)^{\frac{1}{2} v+\frac{1}{4}} P_{n}^{(\mu, v)}(x) \\
& \text { with } x=\frac{s}{\sqrt{q}}, \mu=-\zeta_{-}+\frac{b\left(a+\frac{1}{2}\right)}{\zeta_{-} \sqrt{q}} \text { and } v=-\zeta_{-}-\frac{b\left(a+\frac{1}{2}\right)}{\zeta_{-} \sqrt{q}}
\end{aligned}
$$

\section{The Calculation of energy spectra and wave functions using mapping method through linear transformation of spatial variable}

3.1 Determination of energy spectrum and wave function of deformed modified Poschl-Teller potential using a mapping

By comparing the deformed potential in equation (16a) and non-deformed potential in equation (16b) we obtain the relation of parameters between non-deformed and deformed hyperbolic Poschl-Teller potential given as

$$
\kappa(\kappa-1)+l\left(l+1 \rightarrow \frac{\kappa(\kappa-1)+l(l+1)}{q} \quad \lambda(\lambda+1) \rightarrow \frac{\lambda(\lambda+1)}{q}\right.
$$

The energy spectrum, the first part of wave function and the weight function obtained using NU method for non-deformed hyperbolic Poschl-Teller potential are given as

$$
\begin{aligned}
& E_{n}=-\frac{\hbar^{2}}{2 \mu \gamma^{2}}\left\{\left(\sqrt{\frac{1}{4}+\lambda(\lambda+1)}-\sqrt{\frac{1}{4}+\kappa(\kappa-1)+l(l+1)}-2 n-1\right)^{2}-l(l+1) d_{0}\right\} \\
& \phi=\left(\cosh ^{2} \frac{r}{\gamma}\right)^{-\frac{\sqrt{\lambda(\lambda+1)+\frac{1}{4}}}{4}+\frac{1}{4}}\left(-\sinh ^{2} \frac{r}{\gamma}\right)^{\frac{\sqrt{\kappa(\kappa-1)+\left(l(1)+\frac{1}{4}\right.}}{2}+\frac{1}{4}} \\
& \rho=\left(\cosh ^{2} \frac{r}{\gamma}\right)^{-\sqrt{\lambda(\lambda+1)+\frac{1}{4}}}\left(-\sinh ^{2} \frac{r}{\gamma}\right)^{\sqrt{\kappa(\kappa-1)+l(l+1)+\frac{1}{4}}}
\end{aligned}
$$


By plugging equations (14) and (67) into equations (68), (69), (70) we have

$E_{2}=-\frac{\hbar^{2}}{2 \mu \gamma^{2}}\left\{\left(\sqrt{\frac{1}{4}+\frac{\lambda(\lambda+1)}{q}}-\sqrt{\frac{1}{4}+\frac{\kappa(\kappa-1)+l(l+1)}{q}}-2 n-1\right)^{2}-l(l+1) d_{0}\right\}$

$\phi_{q}=q^{\frac{\sqrt{\frac{\lambda(\lambda+1)}{q}+\frac{1}{4}}}{2}-\frac{1}{2}-\frac{\sqrt{\frac{\kappa(\kappa-1)+l(l+1)}{q}+\frac{1}{4}}}{2}}\left(\cosh _{q}{ }^{2} \frac{r}{\gamma}\right)^{-\frac{\sqrt{\frac{\lambda(\lambda+1)}{q}+\frac{1}{4}}}{2}+\frac{1}{4}}\left(-\sinh _{q}{ }^{2} \frac{r}{\gamma}\right)^{\frac{\sqrt{\frac{\kappa(\kappa-1)+l(l+1)}{q}+\frac{1}{4}}}{2}+\frac{1}{4}}$

and the deformed weight function

$\rho_{q}=q^{\sqrt{\frac{\lambda(\lambda+1)}{q}+\frac{1}{4}}-\sqrt{\frac{\kappa(\kappa-1)+l(l+1)}{q}+\frac{1}{4}}}\left(\cosh _{q}{ }^{2} \frac{r}{\gamma}\right)^{-\sqrt{\frac{\lambda(\lambda+1)}{q}+\frac{1}{4}}}\left(-\sinh ^{2} \frac{r}{\gamma}\right)^{\sqrt{\frac{\kappa(\kappa-1)+l(l+1)}{q}+\frac{1}{4}}}$

The second part of deformed wave function is given as

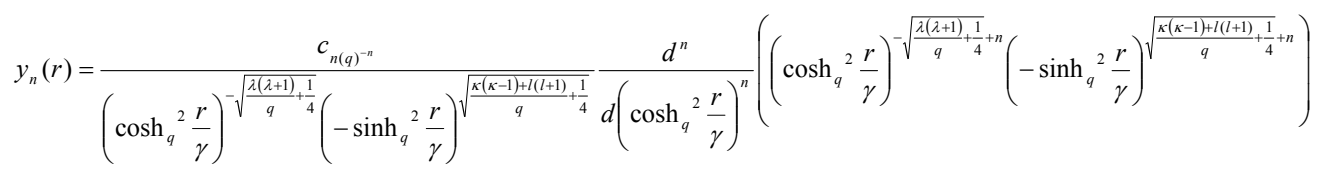

The first wave function and the weight function obtained through a mapping using transformation of spatial variable are in agreement with the result obtained using NU method which are expressed in equations (37), (39).

3.2 Determination of energy spectrum and wave function of hyperbolic Scarf II potentials using a mapping

Secondly, we will determine the energy spectrum and wave functions of q-deformed hyperbolic Scarf II potential with centrifugal term by a transformation of potential parameter. By comparing equations (16a) and (16b) we get the relation of hyperbolic Scarf II's potential parameter between the deformed and non-deformed ones given as

$b^{2}+a(a+1)+l(l+1) \rightarrow \frac{b^{2}+a(a+1)+l(l+1)}{q}$ and $2 b\left(a+\frac{1}{2}\right) \rightarrow 2 b(a+1 / 2) / \sqrt{q}$

The energy spectrum, the first part of wave function and the weight function are given as

$E_{n}=-\frac{\hbar^{2}}{2 m}\left[\alpha^{2}\left[-\sqrt{\frac{\left(b^{2}+a(a+1)+l(l+1)+\frac{1}{4}\right)-\sqrt{\left(b^{2}+a(a+1)+l(l+1)+\frac{1}{4}\right)^{2}-4\left(b\left(a+\frac{1}{2}\right)\right)^{2}}}{2}}+n+\frac{1}{2}\right)^{2}-\alpha^{2} l(l+1) d_{0}\right]$

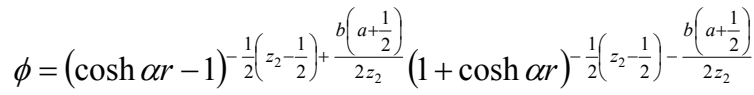

and $\rho=(\cosh \alpha r-1)^{-z_{2}+\frac{b\left(a+\frac{1}{2}\right)}{z_{2}}}(1+\cosh \alpha r)^{-z_{2}-\frac{b\left(a+\frac{1}{2}\right)}{z_{2}}}$

with $z_{2}=\sqrt{\frac{\left(\left(b^{2}+a(a+1)+l(l+1)+\frac{1}{4}\right)-\sqrt{\left(b^{2}+a(a+1)+l(l+1)+\frac{1}{4}\right)^{2}-\left(2 b\left(a+\frac{1}{2}\right)\right)^{2}}\right)}{2}}$

By inserting equation (14) and (75) into equations (76), (77), and (78) we have the energy spectrum, the first part of wave function, and the weight function given as

$E_{n}=-\frac{\hbar^{2}}{2 m}\left[\alpha^{2}\left[-\sqrt{\frac{\left(b^{2}+a(a+1)+l(l+1)+\frac{q}{4}\right)-\sqrt{\left(b^{2}+a(a+1)+l(l+1)+\frac{q}{4}\right)^{2}-4 q\left(b\left(a+\frac{1}{2}\right)\right)^{2}}}{2 q}}+n+\frac{1}{2}\right)^{2}-\alpha^{2} l(l+1) d_{0}\right]$ 


$$
\begin{aligned}
& \phi=q^{\frac{1}{2}\left(z_{2 q}-\frac{1}{2}\right)}\left(\cosh _{q} \alpha r-\sqrt{q}\right)^{-\frac{1}{2}\left(z_{2 q}-\frac{1}{2}\right)+\frac{\frac{b\left(a+\frac{1}{2}\right)}{\sqrt{q}}}{2 z_{2 q}}}\left(\sqrt{q}+\cosh _{q} \alpha r\right)^{-\frac{1}{2}\left(z_{2 q}-\frac{1}{2}\right)-\frac{\frac{b\left(a+\frac{1}{2}\right)}{\sqrt{q}}}{2 z_{2 q}}} \\
& \rho=q^{z_{2 q}}\left(\cosh _{q} \alpha r-\sqrt{q}\right)^{-z_{2 q}+\frac{b\left(a+\frac{1}{2}\right)}{\sqrt{q}}}\left(\sqrt{z_{2 q}}+\cosh _{q} \alpha r\right)^{-z_{2 q}-\frac{b\left(a+\frac{1}{2}\right)}{\sqrt{q}}} \\
& \text { With } z_{z_{2 q}}=\sqrt{\left(\frac{\left.\left(\frac{b^{2}+a(a+1)+l(l+1)}{q}+\frac{1}{4}\right)-\sqrt{\left(\frac{b^{2}+a(a+1)+l(l+1)}{q}+\frac{1}{4}\right)^{2}-\left(\frac{2 b\left(a+\frac{1}{2}\right)}{\sqrt{q}}\right)}\right)}{2}\right.}
\end{aligned}
$$

Equations (80), (81), and (82) are similar to the equations (60), (61), and (62) since equation (57) is the same with equation (83). Therefore the total wave function calculated using a mapping will also be in agreement with the result of direct calculation using NU method.

\section{Conclusion}

The energy spectra and wave functions of deformed modified Poschl-Teller and hyperbolic Scarf potentials are analyzed using NU method and a mapping method. The approximate energy spectra of the two system are expressed in the closed form and the radial wave function are expressed in term of Jacobi polynomials. By a translation of spatial variable the potential parameters of non-deformed potential are mapped into the parameters of the deformed ones, and in turn the energy spectra and wave functions of the non-deformed potentials are mapped into energy spectra and wave functions of the deformed potentials.

\section{References}

Agboola, D.(2011), Dirac equation with spin symmetry for the modified Pöschl-Teller potential in D dimensions, Pramana Journal of Physics, Vol. 76 (6), 875-885

Akpan, I. O., Antia, A. D. \& Ikot, A. N., (2012), Bound-State Solutions of the Klein-Gordon Equation with q-Deformed Equal Scalar and Vector Eckart Potential Using a Newly Improved Approximation Scheme" ISRN High Energy Physics, Vol. 2012, Article ID 798209, 13, doi:10.5402/2012/798209,

Arai, A., (1991), Exactly solvable supersymmetric quantum mechanics. Journal Mathematical Analysis Application 158(63), 63-79.

Ballesteros, A., Civitarese, O.\& Reboiro, M., (2005)Nonstandard q-deformed realizations of the harmonic oscillator, Physics Review C 72, 014305

Bonatsos, D., Daskaloyannis, C. \& Kokkotas, K. (1992), Classical potentials for q-deformed anharmonic oscillators, Physics Review A 45, 6153-6156

Dalarsson, N., (2005), Ab Initio Analytical Approach to Spectral Behavior of Graded Interfaces Incorporating Negative-Index Nanocomposites, Proceeding first International Workshop on Nanoscience \& Nanotechnology IWON, Belgrade, Serbia and Montenegro, November 15 - 18, 2005.

Darareh, M. D. \& Harouni, M. B., (2011), Nonclassical properties of a particle in a finite range trap: the f- deformed quantum oscillator approach, arXiv [quant-ph]:1201.0247v1

Derezi'nski,J. \& Wrochna, M., (2010), Exactly solvable Schr“odinger operators, arXiv [math$p h]: 1009.0541 \mathrm{v} 2$

Dutra, A.S., (2005) Mapping deformed hyperbolic potentials into non-deformed ones, arXiv:quant-ph/0501094v1

Eg rifes, H. Demirhan, D. Büyu"kkılıc, F., (2000), Exact solutions of the Schro"dinger equation for the deformed hyperbolic potential well and the deformed four-parameter exponential type potential, Physics Letters A 275, 229-237 
Eshghi, M., (2012) Dirac-hyperbolic scarf problem including a coulomb-like tensor potential, Acta Scientiarum, Technology Maringá, Vol. 34 (2), 207-215

Greene, R. L. \& Aldrich, C., (1976) "Variational wave functions for a screened Coulomb potential," Physical Review A, Vol. 14 (6), pp. 2363-2366.

Hassan, H., Hoda, Y.B. \& liang-Liang, L. (2012) Approximate Analytical Solutions to the Generalized Poschl-Teller in D Dimensions, Chinese Physics Letter Vol. 29(2), 020303, 1-4

Honusek, M., Vinduśka, M.\& Wagne, V., (1992), Rotational spectra of deformed nuclei and the quantum group SUq (2), Czechoslovak Journal of Physics, Vol. 42 (12), 1337-1344

Ikhdair, S. M. \& Sever, R. (2009) Improved analytical approximation for arbitrary $l$-state solutions of the Schrodinger equation for the Hyperbolic potentials, Annalen der Physik, Vol. 18 (10-11), 747-758

Jia, C S, Sun, Y. Li, Y. (2002), Complexified Pöschl-Teller II potential model, Physics Letters A 305, 231-238.

Nikiforov, A. F.\& Uvarov, V. B. (1998), Special Functions of Mathematical Physics,

Birkhauser, Basel.

Suparmi, (2011) Mekanika Kuantum II, Penerbit Jurusan Fisika UNS, Indonesia

Suparmi, A., Cari, C., Handhika, J., Yanuarief, C. \& Marini, H. (2012) “Approximate Solution of Schrodinger Equation for Modified Poschl-Teller plus Trigonometric Rosen-Morse NonCentral potentials in terms of Finite Romanovski Polynomials", IOSR-Journal of Applied Physics, Vol. 2(2) ISSN 2278-4861, DOI:109790/4861-0224351, 43-51

Sviratcheva, K.D., Bahri, C., Georgieva, A.I. \& Draayer, J.P., (2004), "Physical Significance of q Deformation and Many-Body Interactions in Nuclei," Physical Review Letter 93, 152501.

Spiridonov, V., (1992) Deformed Conformal and Supersymmetric Quantum Mechanics, arXiv:hep- th/9202013v1

Swamy, P. N. (1998), q-Deformed Boson Oscillators And Zero Point Energy, International Journal of Modern Physics B, Vol. 12 (32), World Scientific Publishing Company, 3495-3502

Yasuk, F., Berkdemir, C. \& Berkdemir, A. (2005), "Exact solutions of the Schr"odinger equation with non-central potential by the Nikiforov-Uvarov method," J. Phys. A: Math. Gen., Vol. 38, 6579 
This academic article was published by The International Institute for Science, Technology and Education (IISTE). The IISTE is a pioneer in the Open Access Publishing service based in the U.S. and Europe. The aim of the institute is Accelerating Global Knowledge Sharing.

More information about the publisher can be found in the IISTE's homepage: http://www.iiste.org

\section{CALL FOR PAPERS}

The IISTE is currently hosting more than 30 peer-reviewed academic journals and collaborating with academic institutions around the world. There's no deadline for submission. Prospective authors of IISTE journals can find the submission instruction on the following page: http://www.iiste.org/Journals/

The IISTE editorial team promises to the review and publish all the qualified submissions in a fast manner. All the journals articles are available online to the readers all over the world without financial, legal, or technical barriers other than those inseparable from gaining access to the internet itself. Printed version of the journals is also available upon request of readers and authors.

\section{IISTE Knowledge Sharing Partners}

EBSCO, Index Copernicus, Ulrich's Periodicals Directory, JournalTOCS, PKP Open Archives Harvester, Bielefeld Academic Search Engine, Elektronische Zeitschriftenbibliothek EZB, Open J-Gate, OCLC WorldCat, Universe Digtial Library, NewJour, Google Scholar

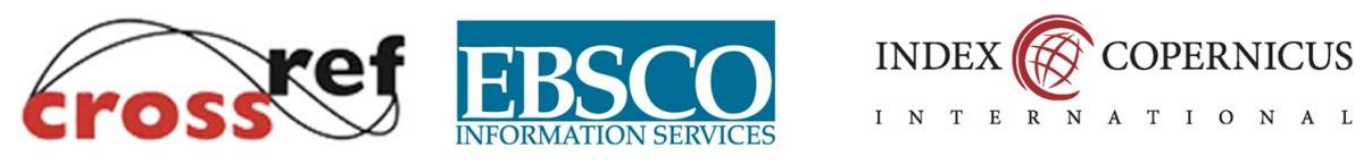

(O) ULRICHSWEB"

JournalTOCs

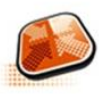

PKP | PUBLIC KNOWLEDGE PROJECT
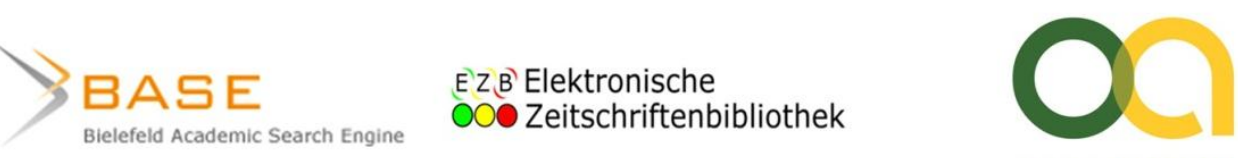

open access
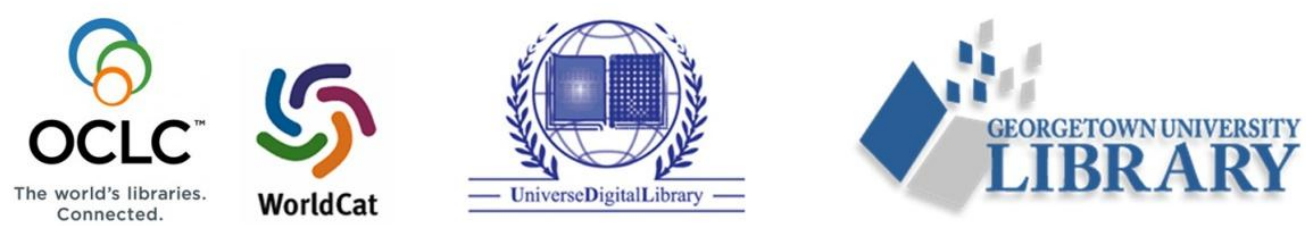